\title{
Impact of Weight Loss on Patients with Locally Advanced Esophageal and Esophagogastric Junction Cancers Treated with Chemoradiotherapy
}

\author{
Jirapath Wiwitkeyoonwong ${ }^{1}$, Chuleeporn Jiarpinitnun ${ }^{2}$, Pitichote Hiranyatheb ${ }^{3}$, \\ Nuttapong Ngamphaiboon ${ }^{1 *}$
}

\begin{abstract}
Introduction: Malnutrition and weight loss are commonly observed in patient with esophageal and esophagogastric junction (EGJ) cancers. Chemoradiotherapy (CRT) is a mainstay of treatment for locally advanced esophageal and EGJ cancers. Impact of weight loss on patients with treated with CRT was not well studied. Methods: Patients with locally advanced esophageal and EGJ cancer who received CRT were identified in our institutional database and allocated into low (LWL) and high (HWL) weight loss groups. HWL was defined as weight loss $>5 \%$ of baseline during CRT. Results: A total of 167 patients were underwent definitive $(n=89)$ or preoperative $(n=78)$ CRT, respectively. HWL was observed in $46 \%$ and $55 \%$ of patients treated with definitive and preoperative CRT, respectively. Cisplatin/5FU regimen used during CRT was a significant predictive factor for weight loss in multivariate analysis (OR 2.07, 95\% CI 1.09-3.94; $\mathrm{p}=0.026$ ). In the definitive CRT group, patients in the HWL group experienced significantly worse overall survival than those in the LWL group (1.2 years vs 1.95 years; $p=0.003$ ). Multivariate analysis revealed that baseline albumin (>3.0 g/dL) was significantly associated with longer OS of definitive CRT patients (HR 2.15, 95\% CI 1.1-4.19; $\mathrm{p}=0.024)$. Tolerability and toxicities during CRT were not statistically different between groups. Conclusion: Significant weight loss during CRT was frequently observed in patients with locally advanced esophageal and EGJ cancers. Baseline hypoalbuminemia was an independent prognostic factor for OS in patients treated with definitive CRT. Nutritional support before and during treatment should be considered to potentially improve patients' outcomes.
\end{abstract}

Keywords: Esophageal cancer- weight loss- chemoradiotherapy- malnutrition- trimodality

Asian Pac J Cancer Prev, 22 (12), 3847-3855

\section{Introduction}

Esophageal and esophagogastric junction (EGJ) cancers are the eighth most common cancers worldwide, the sixth most common cause of cancer related death (Cools-Lartugue et al., 2015) and the tenth most common cancers in Thailand (National cancer Thailand 2018). The two main subtypes of esophageal cancer are esophageal squamous cell carcinoma and adenocarcinoma (Jemal et al., 2011). The squamous cell carcinoma subtype accounts for approximately $90 \%$ of all esophageal cancers worldwide, and in Thailand. (Torre et al., 2015; Tamtai et al., 2017). The survival rates of most patients diagnosed with locally advanced disease range from $40 \%$ to $60 \%$ (Cheng et al., 2012; Di Fiore et al., 2006; Tamtai et al., 2017).

Preoperative chemoradiotherapy (CRT) followed by surgery, known as trimodality, is the standard of care for patients with locally advanced resectable esophageal cancer (Sun, Zhang, and Wu 2014; Shapiro et al. 2015; Tepper et al. 2008; Bedenne et al. 2007). Definitive CRT is employed as the mainstay to achieve good performance of patients with unresectable locally advanced esophageal cancer (Minsky et al .,2002) Progressive dysphagia, initially effecting intake of solids with progression to intake of liquids, is a typical symptomatic presentation of esophageal cancer. Dysphagia can reduce oral intake, and altered nutrient metabolism, associated with systemic inflammation induced by the cancer, can contribute to malnutrition, which is a common comorbidity (Bozzetti et al. 1982). Compared with patients suffering from other cancers, the highest incidence $(78.9 \%)$ of malnutrition is experienced by patients with esophageal and EGJ cancers (Larrea et al., 1992)Weight loss is a more common presenting symptom of esophageal cancer (Daly et al., 2000).

${ }^{1}$ Division of Medical Oncology, Department of Medicine, Faculty of Medicine, Ramathibodi Hospital, Mahidol University, Thailand. ${ }^{2}$ Division of Radiation Oncology, Department of Radiology, Faculty of Medicine, Ramathibodi Hospital, Mahidol University, Thailand. ${ }^{3}$ Division of General Surgery, Department of Surgery, Faculty of Medicine Ramathibodi Hospital, Mahidol University, Thailand.*For Correspondence: nuttapong.nga@mahidol.ac.th 
Before diagnosis, $80 \%$ of patients with esophageal cancer experience $>10 \%$ unintentional weight loss (Larrea et al. 1992). Furthermore, significant weight loss before surgery is associated with shorter 5-year survival (van der Schaaf et al. 2014) and an increase in postoperative complications (Saito et al., 1990 ; Conti, West, and Fitzpatrick 1977). Numerous studies report the effects of weight loss and body mass index (BMI) on the prognosis of patients with esophageal cancer patients who undergo surgery. For example, high BMI at baseline has a significant adverse impact on the long-term survival of patients with esophageal squamous cell carcinoma patients after esophagectomy (Duan et al., 2017). Furthermore, weight loss serves as a prognostic factor for patients with esophageal cancer who undergo upfront surgery and receive adjuvant chemotherapy. Moreover, pretreatment weight loss significantly affects overall survival (Yu et al., 2018). Furthermore, a prospective study from Columbia university reported pre-diagnosis weight loss before diagnosis is associated with the mortality rate of early stage esophageal cancer, particularly for clinical T1 and T2 stages (Crehange et al., 2006).

However, most studies evaluated the association of weight loss with surgery. Impact of weight loss on patients with treated with CRT was not well studied. Therefore, we evaluated the associations between weight loss, treatment-related complications, and survival outcomes of patients with locally advanced esophageal carcinoma treated with CRT.

\section{Materials and Methods}

\section{Study population}

Patients diagnosed with esophageal and esophagogastric junction cancers were treated at Ramathibodi Hospital from January 2011 to the end of June 2019. They were identified through a search of the Ramathibodi Cancer Registry database. We included patients with histologically or cytologically confirmed squamous cell carcinoma, adenocarcinoma, adenosquamous carcinoma, or another specific carcinoma, who underwent CRT with or without surgery. We excluded patients with sarcoma, melanoma, gastrointestinal stromal tumor (GIST), and lymphoma as well as those who underwent surgery without preoperative CRT, had metastatic disease, or those with unavailable medical records. The study was performed according to the principles of the 1964 Declaration of Helsinki. The Ramathibodi Ethics Committee approved the study.

\section{Data collections}

All available medical records were retrospectively reviewed and abstracted. Patients' weights were recorded at the initial visit and defined as weight at diagnosis. Pretreatment weights were measured on the first day of CRT and were recored within 3 days before treatment was terminated. We categorized patients into (1) low weight loss (LWL) and (2) high weight loss (HWL) groups during CRT, defined as the percentage of the difference of weights between baseline and when CRT was termintated, $<5 \%$ and $\geq 5 \%$, respectively, based on previously described in the literature.( $\mathrm{Yu}$ et al., 2018 ;Shen et al., 2013 ;Jiang et al., 2014) BMI, which was defined as the weight $(\mathrm{kg})$ at baseline divided by the square of patients' height(meters) was categorized according to the WHO recommedations for Asian populations ('Appropriate body-mass index for Asian populations and its implications for policy and intervention strategies' 2004). Other information included patients' baseline charactristics, comordities, Eastern Cooperative Oncology Group (ECOG) performance status, stage at diagnosis (AJCC 8th edition), tumor location, histology subtypes, treatment (chemotherapy, radiation,surgery outcome), and complications. Eligible patients were categorized according to the initial aim of treatment (preoperative vs definitive CRT). Only platinum-based plus5FU and carboplatin plus paclitaxel chemotharapy regimens for CRT were allowed. Other nonstandard chemotherapy regimens were excluded. Survival data were extracted from the available medical records. For survival analysis, patients lost to follow-up were matched with the Thai National Death Index.

\section{Treatment complications and outcomes}

Toxicities were reported using the National Cancer Institute's Common Terminology Criteria for Adverse Event (CTCAE) version 5.0. (SERVICES USDOHAH , 2017). Toxicities of interest including hematological toxicites, fatique, acute kidney injury, nausea, and vomiting were recorded. Tolerance to CRT was defined as chemotherapy, radiation interruption, or both, dose reduction $\geq 15 \%$ and early termination of chemotherapy or radiotherapy. The time from completion of CRT to surgery and 90-day postoperative mortality rates were recorded. The pathological response was used to evaluate the efficacy of CRT. A pathological complete response (pCR) was defined as the absence of gross and microscopic tumor tissue from the primary site and lymph nodes upon examination of the surgical specimen.

\section{Statistical analysis}

The primary objective of the study was to evaluate the effect of weight lost during CRT treatment on complications and survival outcomes. Descriptive statistics were used to evaluate patients' characteristics, treatment, complications, and outcomes. Categorical variables are expressed as numbers (percentages). Continuous variables are presented as the mean or median, as appropiate. The chi-square and Fisher's exact tests were used to compare categorical variables, and the Student $t$ test was used to compare continuous variables. Overall surival (OS) was estimated using the Kaplan-Meier method, and datasets were compared using the log-rank test. A Cox proportional hazards model was used to analyze risk factors that affected OS. Predictive factors potentially associated with weight loss during treatment were analyzed using a logistic regression method, and $\mathrm{p}<0.05$ indicates a significant differences. Significant variables identified using univariate analysis were subjected to multivariate analysis. Calculations were performed using Stata version 15.1 (StataCorp, College Station, TX, USA). 
DOI:10.31557/APJCP.2021.22.12.3847

Weight Loss in Patients with Locally Advanced Esophageal Cancer Treated with Chemoradiotherapy

Table 1. Baseline Characteristics and Treatments of Patients Allocated to High Weight Loss and Low Weight Loss Groups

\begin{tabular}{|c|c|c|c|c|c|c|}
\hline \multirow[t]{2}{*}{ Characteristic } & \multicolumn{3}{|c|}{ Preop CRT $(\mathrm{N}=78)(\%)$} & \multicolumn{3}{|c|}{ Definitive CRT (N=89) (\%) } \\
\hline & $\begin{array}{c}\text { LWL }(\mathrm{n}=35) \\
(45 \%)\end{array}$ & $\begin{array}{c}\text { HWL }(\mathrm{n}=43) \\
(55 \%)\end{array}$ & p-value & $\begin{array}{c}\text { LWL }(\mathrm{n}=48) \\
(54 \%)\end{array}$ & $\begin{array}{c}\text { HWL }(\mathrm{n}=41) \\
(46 \%)\end{array}$ & p-value \\
\hline Median Age(range) & $62(47-76)$ & $60(45-81)$ & 0.24 & $62.6(40-83)$ & $62.5(46-84)$ & 0.92 \\
\hline Age $\geq 65$ & $13(37 \%)$ & $10(23 \%)$ & 0.18 & $22(45 \%)$ & $18(44 \%)$ & 0.85 \\
\hline \multicolumn{7}{|l|}{ Sex } \\
\hline Male & $31(89 \%)$ & $40(93 \%)$ & 0.49 & $40(83 \%)$ & $37(90 \%)$ & 0.34 \\
\hline Female & $4(11.4 \%)$ & $3(7 \%)$ & & $8(17 \%)$ & $4(10 \%)$ & \\
\hline \multicolumn{7}{|l|}{ Smoking } \\
\hline Never & $4(12 \%)$ & $4(9 \%)$ & 0.69 & $10(22 \%)$ & $9(22 \%)$ & 1 \\
\hline ever & $29(88 \%)$ & $39(91 \%)$ & & $35(78 \%)$ & $31(78 \%)$ & \\
\hline Mean $(\mathrm{pack} / \mathrm{yr}+/=\mathrm{SD})$ & $25.5+3.7$ & $20.9+2.6$ & 0.31 & $21.3+2.4$ & $30.7+4.2$ & 0.048 \\
\hline \multicolumn{7}{|l|}{ ECOG } \\
\hline 0 & $13(37 \%)$ & $16(37 \%)$ & 0.71 & $7(15 \%)$ & $9(22 \%)$ & 0.68 \\
\hline 1 & $21(60 \%)$ & $27(63 \%)$ & & $38(79 \%)$ & $30(73 \%)$ & \\
\hline 2 & $1(3 \%)$ & 0 & & $3(6 \%)$ & $2(5 \%)$ & \\
\hline \multicolumn{7}{|l|}{ Underlying } \\
\hline COPD & 0 & $1(2 \%)$ & 1 & $2(4 \%)$ & $2(5 \%)$ & 1 \\
\hline HT & $9(26 \%)$ & $15(35 \%)$ & 0.46 & $11(23 \%)$ & $9(22 \%)$ & 1 \\
\hline $\mathrm{DM}$ & $4(11 \%)$ & $4(9.3 \%)$ & 1 & $4(8 \%)$ & $4(10 \%)$ & 1 \\
\hline CAD & $1(3 \%)$ & $1(2 \%)$ & 1 & $3(6 \%)$ & $5(12 \%)$ & 0.46 \\
\hline Second malignancy & $1(3 \%)$ & $5(12 \%)$ & 0.22 & $10(21 \%)$ & $6(15 \%)$ & 0.45 \\
\hline Baseline Mean Weight $(\mathrm{kg})(+/-\mathrm{SD})$ & $55.6+1.8$ & $57+1.4$ & 0.43 & $52.3+1.4$ & $58.7+1.5$ & 0.045 \\
\hline Mean BMI & $20.4+.6$ & $21+.4$ & 0.31 & $19+0.5$ & $21+0.5$ & 0.012 \\
\hline$\leq 18.5$ & $9(26 \%)$ & $9(21 \%)$ & 0.87 & $22(46 \%)$ & $6(15 \%)$ & 0.007 \\
\hline $18.6-23$ & $17(49 \%)$ & $23(53 \%)$ & & $18(38 \%)$ & $24(58 \%)$ & \\
\hline$>23$ & $9(26 \%)$ & $11(26 \%)$ & & $8(17 \%)$ & $11(27 \%)$ & \\
\hline \multicolumn{7}{|l|}{ Clinical T stage } \\
\hline $1-2$ & $8(24 \%)$ & $6(14 \%)$ & 0.28 & $14(29 \%)$ & $8(20 \%)$ & 0.29 \\
\hline $3-4$ & $26(76 \%)$ & $37(86 \%)$ & & $34(71 \%)$ & $33(80 \%)$ & \\
\hline \multicolumn{7}{|l|}{ Clinical N stage } \\
\hline Negative & $4(12 \%)$ & $14(33 \%)$ & 0.032 & $10(21 \%)$ & $5(12 \%)$ & 0.28 \\
\hline Positive & $30(88 \%)$ & $29(67 \%)$ & & $38(79 \%)$ & $36(88 \%)$ & \\
\hline \multicolumn{7}{|l|}{ Clinical Stage (AJCC 8th ) } \\
\hline I-II & $10(29 \%)$ & $16(37 \%)$ & 0.47 & $12(25 \%)$ & $6(15 \%)$ & 0.225 \\
\hline III & $24(71 \%)$ & $27(63 \%)$ & & $36(75 \%)$ & $35(85 \%)$ & \\
\hline \multicolumn{7}{|l|}{ Histology subtype } \\
\hline Squamous cell & $34(97 \%)$ & $37(86 \%)$ & 0.19 & $45(94 \%)$ & $28(90 \%)$ & 0.128 \\
\hline Adenocarcinoma & 0 & $3(7 \%)$ & & $2(4 \%)$ & 0 & \\
\hline Other & $1(3 \%)$ & $3(7 \%)$ & & $1(2 \%)$ & $4(10 \%)$ & \\
\hline \multicolumn{7}{|l|}{ Primary Tumor location } \\
\hline proximal & $2(6 \%)$ & $1(2 \%)$ & 0.4 & $25(52 \%)$ & $18(44 \%)$ & 0.65 \\
\hline Middle & $21(60 \%)$ & $22(51 \%)$ & & $18(38 \%)$ & $19(46 \%)$ & \\
\hline Distal & $12(34 \%)$ & $17(40 \%)$ & & $4(8 \%)$ & $4(10 \%)$ & \\
\hline EGJ & 0 & $3(7 \%)$ & & $1(2 \%)$ & 0 & \\
\hline \multicolumn{7}{|l|}{ Feeding route pre CRT } \\
\hline Oral & $10(29 \%)$ & $11(26 \%)$ & 0.77 & $14(29 \%)$ & $8(20 \%)$ & 0.29 \\
\hline Feeding tube & $25(71 \%)$ & $32(74 \%)$ & & $34(71 \%)$ & $33(80 \%)$ & \\
\hline
\end{tabular}


Table 1. Continued

\begin{tabular}{|c|c|c|c|c|c|c|}
\hline \multirow[t]{2}{*}{ Characteristic } & \multicolumn{3}{|c|}{ Preop CRT $(\mathrm{N}=78)(\%)$} & \multicolumn{3}{|c|}{ Definitive CRT (N=89) (\%) } \\
\hline & $\begin{array}{c}\text { LWL }(\mathrm{n}=35) \\
(45 \%)\end{array}$ & $\begin{array}{c}\text { HWL }(\mathrm{n}=43) \\
(55 \%)\end{array}$ & p-value & $\begin{array}{c}\text { LWL (n=48) } \\
(54 \%)\end{array}$ & $\begin{array}{c}\text { HWL }(\mathrm{n}=41) \\
(46 \%)\end{array}$ & p-value \\
\hline \multicolumn{7}{|l|}{ Baseline Albumin (g/dL) } \\
\hline Mean (+/-SD) & $3.5+0.1$ & $3.65+.08$ & 0.25 & $3.36+.08$ & $3.24+0.12$ & 0.46 \\
\hline Median (range) & $3.65(2.43-4.4)$ & $3.57(2.47-4.9)$ & & $3.37(2.4-4.3)$ & $3.24(1.2-4.8)$ & \\
\hline$>3.0$ & $48(81 \%)$ & $59(82 \%)$ & 0.93 & $28(82 \%)$ & $23(70 \%)$ & 0.22 \\
\hline$\leq 3.0$ & $11(19 \%)$ & $13(18 \%)$ & & $6(18 \%)$ & $10(30 \%)$ & \\
\hline \multicolumn{7}{|l|}{ Chemotherapy regimen for CRT } \\
\hline Carboplatin/paclitaxel & $28(80 \%)$ & $27(63 \%)$ & 0.097 & $28(58 \%)$ & $14(34 \%)$ & 0.023 \\
\hline Platinum/5-FU & $7(20 \%)$ & $16(37 \%)$ & & $20(42 \%)$ & $27(66 \%)$ & \\
\hline \multicolumn{7}{|l|}{ Mean actual dose } \\
\hline Cisplatin $\left(\mathrm{mg} / \mathrm{m}^{2}\right)$ & $151.4(+3.9)$ & $134.4(+30.1)$ & 0.155 & $137.2(+31.8)$ & $141.3(+46.5)$ & 0.76 \\
\hline Carboplatin(AUC) & $10.3(+1.7)$ & $10.1(+2.4)$ & 0.6 & $9.8(+2.8)$ & $9.8(+2.3)$ & 0.95 \\
\hline $5-\mathrm{FU}\left(\mathrm{mg} / \mathrm{m}^{2}\right)$ & $6857(+1069)$ & $6562(+1410)$ & 0.63 & $6530(+1531)$ & $6631(+1584)$ & 0.83 \\
\hline Paclitaxel $\left(\mathrm{mg} / \mathrm{m}^{2}\right)$ & $251.4(+55.9)$ & $239.4(+59.5)$ & 0.49 & $236.8(+87.8)$ & $254.3(+70.5)$ & 0.52 \\
\hline \multicolumn{7}{|l|}{ Radiotherapy technique } \\
\hline 3D-CRT & $31(88.6 \%)$ & $40(93 \%)$ & 0.83 & $32(66.7 \%)$ & $26(63.4 \%)$ & 0.42 \\
\hline 3D+IMRT/VMAT & $3(8.6 \%)$ & $2(4.6 \%)$ & & $7(14.6 \%)$ & $10(24.4 \%)$ & \\
\hline IMRT/VMAT & $1(2.9 \%)$ & $1(2.3 \%)$ & & $9(18.7 \%)$ & $5(12.2 \%)$ & \\
\hline Mean planned dose of $\mathrm{RT} \pm \mathrm{SD}$ (Gy) & $50.05(+3.6)$ & $49.3(+4.5)$ & 0.43 & $56(+5.2)$ & $56(+6.2)$ & 0.97 \\
\hline \multicolumn{7}{|l|}{ Mean actual dose of $\mathrm{RT} \pm \mathrm{SD}$} \\
\hline$\leq 45 \mathrm{~Gy}$ & $3(8.6 \%)$ & $4(9.3 \%)$ & 0.04 & $2(4.2 \%)$ & $2(4.9 \%)$ & 0.045 \\
\hline $45.1-50.3 \mathrm{~Gy}$ & $5(14.3 \%)$ & $17(39.5 \%)$ & & $1(2.1 \%)$ & $7(17.1 \%)$ & \\
\hline$\geq 50.4 \mathrm{~Gy}$ & $27(77.1 \% \%)$ & $22(51.2 \%)$ & & $45(93.8 \%)$ & $32(78 \%)$ & \\
\hline Median Duration of RT (days) (range) & $40.4(+5.0)$ & $39.1(+9.3)$ & 0.45 & $46.75(+14.7)$ & $41.4(+8.3)$ & 0.043 \\
\hline
\end{tabular}

\section{Results}

\section{Patients' characteristics}

Among 332 patients with esophageal or EGJ cancer, 167 patients were included according to the criteria listed in Supplement 1. Patients were categorized into preoperative CRT $(\mathrm{n}=78)$ and definitive CRT $(\mathrm{n}=89)$ groups. Baseline characteristics of patients treated preoperatively with definitive CRT are listed in Table 1. In the preoperative CRT group, 35 patients $(45 \%)$ experienced weight loss $<5 \%$ (LWL), and 43 patients (55\%) experienced weight loss $\geq 5 \%$ (HWL). Most patients were males with good ECOG performance $(0-1)$. Moreover, baseline albumin levels were not significantly different $(80 \%$, albumin $\geq 30 \mathrm{~g} / \mathrm{dl})$. The baseline values of the two groups were well balanced, except $\mathrm{N}$ staging. The LWL group included significantly more patients with clinical node-positive disease ( $88 \%$ vs $67 \%, \mathrm{p}=0.032$ ). Compared with the HWL group, patients in the LWL group were mainly administered carboplatin/paclitaxel, although the difference between groups was not significant.

Significant differences were observed between BMI and weight upon diagnosis of patients treated with definitive CRT. The mean baseline weight of the LWL group was significantly lower than that of the HWL group ( $52 \mathrm{vs} 59 \mathrm{~kg}, \mathrm{p}=0.045$ ). Patients with LWL had significant more proportion of under-weight by baseline BMI when compared with HWL patients $(\mathrm{p}=0.007)$. Most patients in the LWL group received the carboplatin/paclitaxel regimen (58\%), while those in the HWL group were mainly treated with the platinum $/ 5 \mathrm{FU}$ regimen $(66 \%)$ during CRT ( $\mathrm{p}=0.023)$.

Univariate and multivariate analyses with odd ratios (ORs) were used to identify predictive factors of weight loss in patients with locally advanced esophageal cancer (Supplement 2). Univariate analysis revealed that complications before treatment commenced, such as aspiration pneumonia, tracheoesophageal fistula, upper airway obstruction, and infected prophylatic feeding tube were significantly associated with weight loss during treatment (OR 3.25, 95\% CI 1.00-10.52, $\mathrm{p}=0.049$ ). However, these variables were not significant in multivariate analysis (OR 2.98, 95\% CI 0.90-9.84, $\mathrm{p}=0.072$ ). Only the concurrent cisplatin $/ 5 \mathrm{FU}$ chemotherapy regimen with CRT was a significant predictive factor for weight loss in univariate (OR 2.18, 95\% CI 1.16-4.07, $\mathrm{p}=0.015$ ) and multivariate (OR 2.07, 95\% CI 1.09-3.94; $\mathrm{p}=0.026$ ) analyses.

\section{Impact of weight loss on survival outcomes}

Figure 1 shows that the median OS of patients treated with definitive CRT in the HWL group was significantly shorter than that of the LWL group (1.2 years vs 1.95 years, HR 1.76, 95\% CI 1.06-3.01, $\mathrm{p}=0.003$ ). Median diseasefree survival (DFS) of patients in the HWL group was shorter compared with that of the LWL group ( 0.7 years 
vs 1.3 years, HR $1.81,95 \%$ CI $1.08-3.03, \mathrm{p}=0.022)$. In contrast, there were no significant differences between median OS and DFS of patients treated with preoperative CRT who had low or high weight loss (Figure 2).

Univariate analysis of patients treated with definitive CRT demonstrated that clinical T staging, pretreatment albumin levels, weight loss during treatment, and baseline BMI were significant prognostic factors for OS (Table 3). In contrast, only the pretreatment albumin level was a significant prognostic factor for OS $(\mathrm{HR}=2.15,95 \% \mathrm{CI}$ $1.1-4.19, \mathrm{p}=0.024)$. Univariate and multivariate analyses of patients treated with preoperative CRT revealed that surgery was the only significant prognostic factor for OS $(\mathrm{HR}=0.54,95 \%$ CI $0.3-0.93, \mathrm{p}=0.03)$, although weight loss was not.

There were no significant differences in tolerability and toxicities of interest of patients with esophageal cancer who underwent CRT in preoperative or definitive settings (Table 3). The numbers of all-grade toxicities were higher in the HWL group in both treatment settings. However, the effects of dose reduction of chemotherapy were more prominent in the HWL group treated with preoperative CRT $(18.6 \%$ vs $5.7 \%, \mathrm{p}=0.09)$.

Among patients who received preoperative CRT (Supplement 3), 21 (60\%) in the LWL group underwent surgery more frequently than those in the HWL group $(40 \%)$, although the difference was not significant. Furthermore, the mean time from CRT to surgery of the LWL group was shorter ( 94 days vs 142 days, $p=0.235$ ), and the LWL group achieved a higher pCR rate than the HWL group ( $58 \%$ vs $31 \%, \mathrm{p}=0.115)$.

\section{Discussion}

To our knowledge, this study is the first to report the impact of weight loss during CRT by patients with esophageal and EGJ cancers, with focus on those with unresectable locally advanced esophageal cancer. In this study,we observed frequent and significant weight loss during chemoradiotherapy in definitive and preoperative settings of approximately $50 \%$ of patients with locally advanced esophageal cancer, although approximately $75 \%$ of patients underwent prophylactic tube feeding before CRT. These findings demonstrate significant malnutrition

Table 2. Univariate and Multivariate Cox Regression for Survival Analysis

\begin{tabular}{|c|c|c|c|c|c|c|c|c|}
\hline \multirow{3}{*}{ Factor } & \multicolumn{4}{|c|}{ Pre-operative CRT } & \multicolumn{4}{|c|}{ Definitive CRT } \\
\hline & \multicolumn{2}{|c|}{ Univariate } & \multicolumn{2}{|c|}{ Multivariate } & \multicolumn{2}{|c|}{ Univariate } & \multicolumn{2}{|c|}{ multivariate } \\
\hline & $\begin{array}{c}\mathrm{HR} \\
(95 \% \mathrm{CI})\end{array}$ & $\mathrm{P}$ value & $\begin{array}{c}\mathrm{HR} \\
(95 \% \mathrm{CI})\end{array}$ & $\mathrm{P}$ value & $\begin{array}{c}\mathrm{HR} \\
(95 \% \mathrm{CI})\end{array}$ & $P$ value & $\begin{array}{c}\mathrm{HR} \\
(95 \% \mathrm{CI})\end{array}$ & $P$ value \\
\hline AGE & 1.74 & 0.064 & - & - & 0.92 & 0.75 & - & - \\
\hline$<65$ vs $\geq 65$ & $(0.96-3.13)$ & & & & $(0.54-1.55)$ & & & \\
\hline Sex & 0.63 & 0.38 & - & - & 0.47 & 0.076 & - & - \\
\hline Male vs Female & $(0.23-1.75)$ & & & & $(0.19-1.18)$ & & & \\
\hline Stage & & & - & - & & & & \\
\hline T1-2 vs T3-4 & $\begin{array}{c}1.2 \\
(0.60-2.39)\end{array}$ & 0.6 & & & $\begin{array}{c}2.54 \\
(1.24-5.19)\end{array}$ & 0.01 & $\begin{array}{c}1.77 \\
(0.79-3.97)\end{array}$ & 0.166 \\
\hline N0 vs N+ & $\begin{array}{c}1.23 \\
(0.65-2.3)\end{array}$ & 0.186 & & & $\begin{array}{c}1.55 \\
(0.70-3.43)\end{array}$ & 0.28 & & \\
\hline \multicolumn{9}{|l|}{ pretreatment BMI } \\
\hline $18.5-22.99$ vs $\leq 18.5$ & $\begin{array}{c}1.69 \\
(0.90-3.17)\end{array}$ & 0.29 & - & - & $\begin{array}{c}1.46 \\
(0.81-2.61)\end{array}$ & 0.21 & - & - \\
\hline $18.5-22.99$ vs $\geq 23$ & $\begin{array}{c}1.3 \\
(0.68-2.5)\end{array}$ & 0.26 & & & $\begin{array}{c}1.01 \\
(0.50-2.04)\end{array}$ & 0.97 & & \\
\hline $\begin{array}{l}\text { Albumin baseline } \\
>3.0 \mathrm{vs} \leq 3.0 \mathrm{~g} / \mathrm{dL}\end{array}$ & $\begin{array}{c}1.14 \\
(0.51-2.56)\end{array}$ & 0.75 & - & - & $\begin{array}{c}2.37 \\
(1.25-4.5)\end{array}$ & 0.008 & $\begin{array}{c}2.15 \\
(1.10-4.19)\end{array}$ & 0.024 \\
\hline $\begin{array}{l}\text { Surgery } \\
\text { No vs Yes }\end{array}$ & $\begin{array}{c}0.577 \\
(0.33-0.99)\end{array}$ & 0.046 & $\begin{array}{c}0.54 \\
(0.30-0.93)\end{array}$ & 0.03 & N/A & - & - & - \\
\hline \multicolumn{9}{|c|}{ Concomitant chemotherapy regimen } \\
\hline $\begin{array}{l}\text { Carboplatin/paclitaxel } \\
\text { vs Cisplatin/5FU }\end{array}$ & $\begin{array}{c}0.856 \\
(0.47-1.54)\end{array}$ & 0.605 & - & - & $\begin{array}{c}0.84 \\
(0.52-1.50)\end{array}$ & 0.65 & - & - \\
\hline \multicolumn{9}{|l|}{ Actual Dose of $\mathrm{RT} \pm \mathrm{SD}$} \\
\hline$\geq 45 \mathrm{~Gy}$ & $\begin{array}{c}1.23 \\
(0.71-2.13)\end{array}$ & 0.449 & - & - & $\begin{array}{c}0.68 \\
(0.21-2.2)\end{array}$ & 0.549 & - & - \\
\hline \multicolumn{9}{|c|}{ Weight loss during treatment } \\
\hline LWL vs HWL & $\begin{array}{c}0.885 \\
(0.52-1.5)\end{array}$ & 0.655 & $\begin{array}{c}0.75 \\
(0.43-1.30)\end{array}$ & 0.317 & $\begin{array}{c}1.79 \\
(1.06-3.01)\end{array}$ & 0.028 & $\begin{array}{c}1.43 \\
(0.76-2.69)\end{array}$ & 0.258 \\
\hline
\end{tabular}



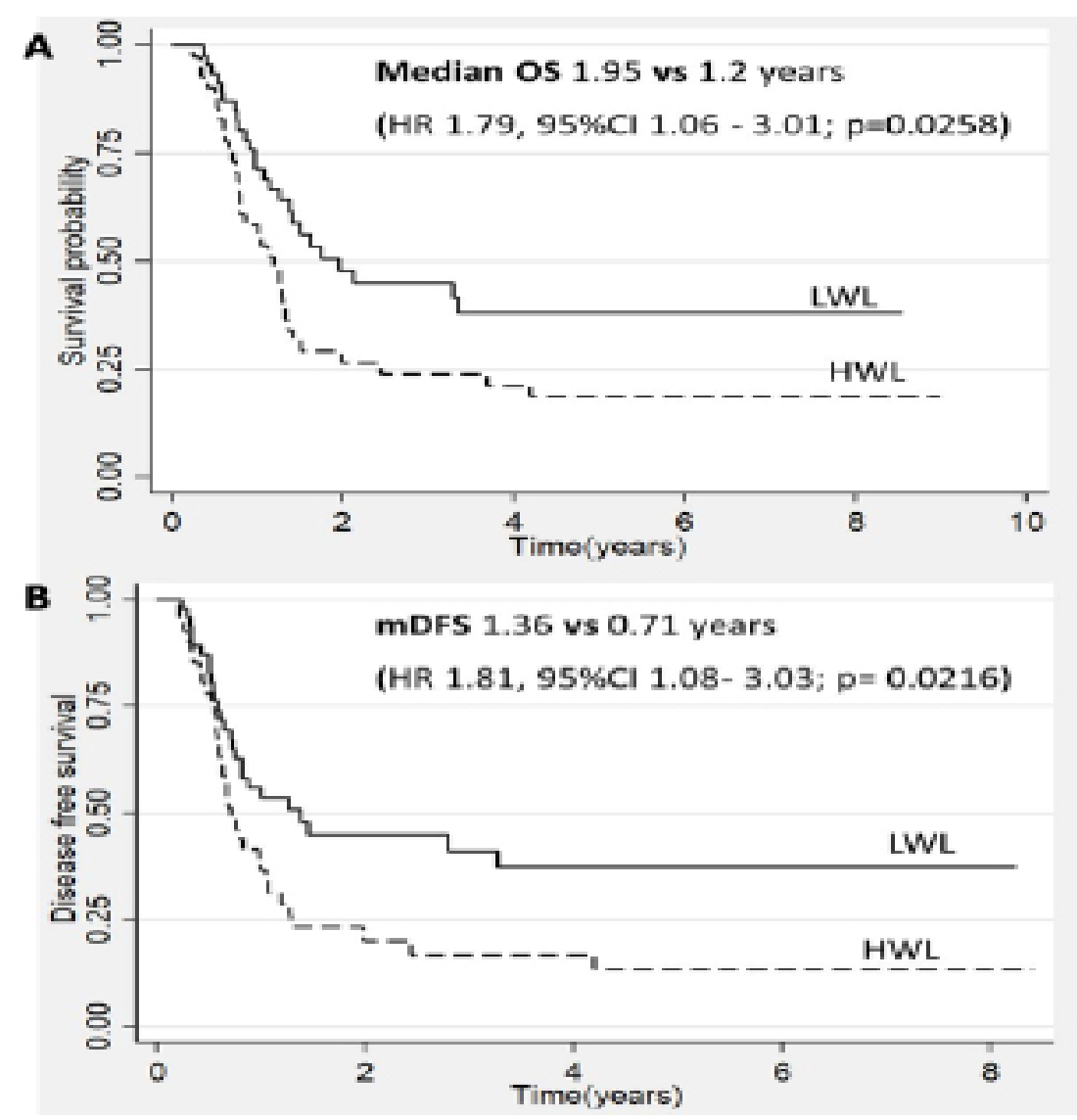

Figure 1. Overall Survival (A), and Disease Free Survival (B) of Patients with Locally Advanced Esophageal Cancer who Received Definitive CRT
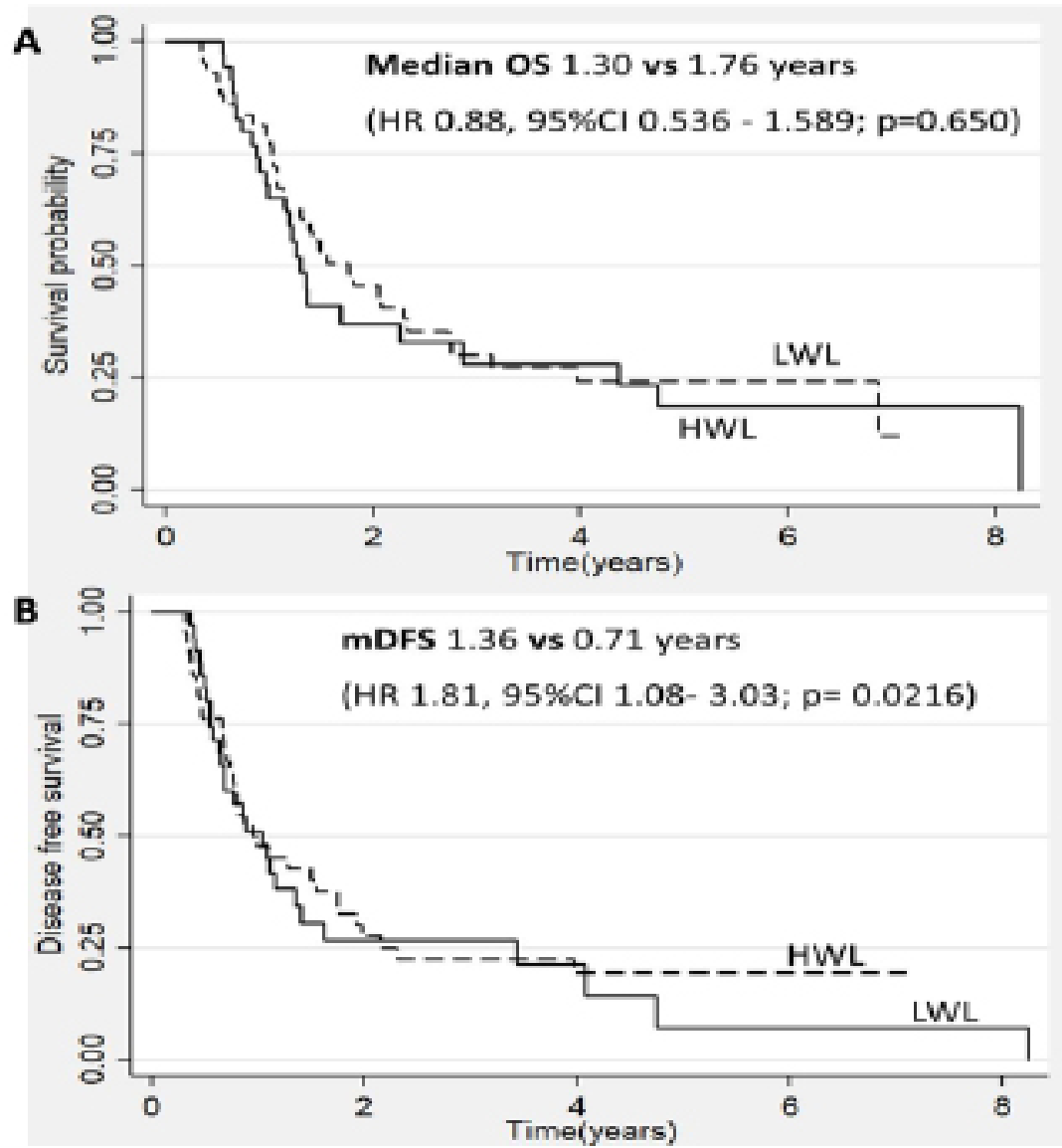

Figure 2. Overall Survival (A) and Disease Free Survival (B) of Patients with Locally Advanced Esophageal Cancer who Received Preoperative CRT 
DOI:10.31557/APJCP.2021.22.12.3847

Weight Loss in Patients with Locally Advanced Esophageal Cancer Treated with Chemoradiotherapy

Table 3. Tolerability and Acute Complications during CRT

\begin{tabular}{|c|c|c|c|c|c|c|}
\hline \multirow[t]{2}{*}{ Complication } & \multicolumn{3}{|c|}{ Pre-op CRT } & \multicolumn{3}{|c|}{ Definitive CRT } \\
\hline & $\begin{array}{c}\operatorname{LWL}(\mathrm{n}=35) \\
(45 \%)\end{array}$ & $\begin{array}{c}\text { HWL }(\mathrm{n}=43) \\
(55 \%)\end{array}$ & p-value & $\begin{array}{c}\operatorname{LWL}(\mathrm{n}=48) \\
(54 \%)\end{array}$ & $\begin{array}{c}\operatorname{HWL}(\mathrm{n}=41) \\
(46 \%)\end{array}$ & $\mathrm{p}$-value \\
\hline \multicolumn{7}{|l|}{ Treatment interruption } \\
\hline Chemotherapy & $14(40 \%)$ & $13(30.2 \%)$ & 0.367 & $22(45.8 \%)$ & $16(39 \%)$ & 0.52 \\
\hline Radiation & $2(5.7 \%)$ & $2(4.6 \%)$ & 0.83 & $2(4.2 \%)$ & $3(7.3 \%)$ & 0.52 \\
\hline Chemotherapy Dose reduction & $2(5.7 \%)$ & $8(18.6 \%)$ & 0.09 & $12(25 \%)$ & $7(17.1 \%)$ & 0.44 \\
\hline Termination of Chemotherapy & $1(2.9 \%)$ & $6(14 \%)$ & 0.122 & $10(20.8 \%)$ & $3(7.3 \%)$ & 0.13 \\
\hline Termination of radiotherapy & 0 & $2(4.6 \%)$ & 0.499 & $2(4.2 \%)$ & $2(4.9 \%)$ & 1 \\
\hline \multicolumn{7}{|l|}{ Overall adverse event } \\
\hline All grade & $29(82.9 \%)$ & $37(86.1 \%)$ & 0.698 & $40(83.3 \%)$ & $37(90.2 \%)$ & 0.34 \\
\hline Gr 3- 4 & $5(14.3 \%)$ & $10(23.3 \%)$ & 0.32 & $14(29.2 \%)$ & $14(34.1 \%)$ & 0.61 \\
\hline \multicolumn{7}{|l|}{ Anemia } \\
\hline All grade & $7(20 \%)$ & $15(34.8 \%)$ & 0.15 & $15(31.3 \%)$ & $20(48.8 \%)$ & 0.091 \\
\hline Gr 3-4 & $1(2.9 \%)$ & $2(4.7 \%)$ & 1 & $2(4.1 \%)$ & $4(9.8 \%)$ & 0.408 \\
\hline \multicolumn{7}{|l|}{ Thrombocytopenia } \\
\hline All grade & $2(5.7 \%)$ & $1(2.3 \%)$ & 0.44 & $1(2.08 \%)$ & $2(4.9 \%)$ & 0.593 \\
\hline Gr 3-4 & $1(2.9 \%)$ & $1(2.3 \%)$ & 1 & $1(2.08 \%)$ & $1(2.4 \%)$ & 1 \\
\hline \multicolumn{7}{|l|}{ Neutropenia } \\
\hline All grade & $5(4.9 \%)$ & $6(6.1 \%)$ & 0.967 & $11(22.9 \%)$ & $5(12.2 \%)$ & 0.19 \\
\hline Gr 3-4 & $1(2.9 \%)$ & $6(23.9 \%)$ & 0.122 & $5(10.42 \%)$ & $2(4.9)$ & 0.445 \\
\hline \multicolumn{7}{|l|}{ Febrile neutropenia } \\
\hline Gr 3-4 & 0 & $3(7.1 \%)$ & 0.111 & $1(1 \%)$ & $1(0.9 \%)$ & 0.91 \\
\hline \multicolumn{7}{|l|}{ Nausea/vomiting } \\
\hline All grade & $8(22.9 \%)$ & $16(37.2 \%)$ & 0.17 & $10(20.8 \%)$ & $13(31.7 \%)$ & 0.24 \\
\hline Gr 3-4 & $1(2.9 \%)$ & $3(7.0 \%)$ & 0.62 & 0 & 0 & \\
\hline \multicolumn{7}{|l|}{ Fatigue } \\
\hline All grade & $14(40.0 \%)$ & $17(39.5 \%)$ & 0.78 & $17(35.4 \%)$ & $11(26.8 \%)$ & 0.385 \\
\hline Gr 3-4 & $1(2.9 \%)$ & $2(4.7 \%)$ & 1 & $2(4.2 \%)$ & $2(4.9 \%)$ & 0.87 \\
\hline \multicolumn{7}{|l|}{ Acute kidney injury } \\
\hline All grade & 0 & $4(9.3)$ & 0.064 & $3(6.2 \%)$ & $2(4.9 \%)$ & 1 \\
\hline Gr 3-4 & 0 & $1(2.3 \%)$ & 1 & 0 & 0 & \\
\hline
\end{tabular}

of patients with locally advanced esophageal cancer. Several studies defined unintentionally high weight loss defined by weight loss $\geq 5 \%$ of body weight, which was similar to the weight loss cut-off using in ours study (Yu et al., 2018 ;Shen et al., 2013 ;Jiang et al., 2014).

For instance, half of patients with esophageal cancer, who received CRT in any setting, experienced $\geq 5 \%$ weight loss during CRT, which was consistent with other studies (Jiang et al., 2014). Furthermore, two studies reported that weight loss in esophageal cancer was significantly associated with poor prognosis (Yu et al., 2018 ; Gu et al., 2019) . Unlike our study, these studies evaluated pretreatment weight loss and BMI in patients undergoing upfront radical surgery without preoperative CRT. Considering the current standard practice for patients with resectable locally advanced esophageal cancer, their results may not be applicable to patients with esophageal cancer who undergo trimodal therapy.

The analysis of the definitive CRT group revealed that median OS was significantly shorter if patients were unable to maintain body weight during treatment. The median OS of the HWL group was comparable to that reported by the RTOG 85-01 study, in which the median OS of patients with locally advanced esophageal cancer patients who received definitive CRT was 12.5 months (Cooper et al., 1999). Patients who cannot maintain body weight during CRT may experience a higher frequency of grade 3-4 CRT-related toxicities such as mucositis and dysphagia as well as hematological toxicity (Jiang et al., 2014). These adverse events may lead to interruption or termination of CRT, which likley contributes to poor treatment outcomes (Huhmann and Cunningham., 2005). We found that the baseline pretreatment albumin level was an independent prognostic factor of OS for such patients. This finding is consistent with previous studies showing that the serum albumin level is an independent prognostic factor for mortality, surgical complications, and outcomes of patients ] with esophageal cancer (Khan, Bangash, and Sadiq., 2010 ;Wu et al., 2015).

Thus, the serum albumin level may serve as a better 
surrogate marker for the nutritional status compared with weight loss. Several studies suggest that the prognostic nutritional index in esophageal cancer comprising the serum albumin level, the neutrophil-to-lymphocyte ratio, total lymphocyte count, or BMI were associated with longterm outcomes of patients with esophageal cancer (Zhang et al., 2017 ;Nakatani et al. 2017). And low prognostic nutrional index is signficantly associated with shorter overall survival (Zhang et al., 2017 ; Liu, Shi and Chen.,2019). On the other hand, in multivariate analysis, weight loss during treatment was not significantly associated with survival outcomes. This inconsistent finding was possibly resulted from a small sample size. Moreover, the patients in our study might have experienced significant weight loss before the diagnosis of esophageal cancer. The absence of data of patient's baseline body weight before diagnosis was a consequence of the retrospective nature of the study and may explain the inconsistency between OS and the impacts of weight loss and baseline serum albumin level.

However, here we were unable to detect a correlation between the baseline serum albumin level and weight loss during CRT. Weight loss was not significantly associated with survival of the preoperative CRT group. This inconsistency may be partially explained by our observation that approximately $50 \%$ of patients did not undergo surgery as initially planned. This may be related to a patient's decision, delayed recovery, or poor nutritional status after completion of CRT. We did observe a trend for patients in the HWL group to undergo fewer surgeries than those in the LWL group (39.5\% vs $60 \%$, respectively; $p=0.072$ ) and a longer time from the completion of CRT to surgery (143 days vs 95 days, respectively; $\mathrm{p}=0.235$ ).

In the preoperative CRT group, weight loss during CRT was not an independent prognostic factor for OS in multivariate analysis, whereas the pre-CRT baseline serum albumin level was not significantly associated with OS in univariate analysis (Table 2). We found that surgical resection was a significant independent prognostic facto for OS. This finding is inconsistent with the results of the phase III FFCD 9102 study that evaluted preoperative CRT followed by surgery vs definitive CRT for resectable locally advanced squanous cell carinoma of the thoracic esophagus (Bedenne et al., 2007). The FFCD 9102 study did not find a benefit for performing surgery after CRT. However, this study was prematurely terminated because of slow accrual and was considered statistically underpowered. Thus, the benefit of surgery in patients with locally advanced esophageal cancer remains to be established. Our study had several limitations. Caloric intake of patients during CRT was not reported in our study due to limitation of retrospective nature although $70-80 \%$ of patients in our study had feeding tube pre-CRT. Data for nutritional markers such as prealbumin levels, daily caloric intake, baseline dysphagia, and body weight before symptoms of esophageal cancer appeared were limited because of the retrospective nature of the study. The relatively small size of each treatment group may lead to inadequate statistical power required to evaluate certain subgroups administered different treatments, such as chemotherapy and radiation techniques and dosing. Therefore, a prospective study of a larger sample size is required to validate these findings.

In summary, significant weight loss during CRT in definitive and preoperative settings was frequently observed in approximately $50 \%$ of patients with locally advanvced esophageal cancer. Significant weight loss was asscociated with poor OS of patients treated with definitive CRT. However, weight loss during CRT was not an independent prognostic factor for OS in definitive and preoperative settings, whereas the baseline serum albumin level was an independent prognositic factor for OS in patients with locally advanced unresectable esophageal cancer treated with definitive CRT. Nutritional support before and during treatment of locally advanced esophageal cancer should be considered to potentially improve outcomes.

\section{Author Contribution Statement}

All authors have read and approved the manuscript. Study concepts: JW, NN. Study design: JW, NN. Data acquisition: JW, CJ, PH. Data analysis and interpretation: JW, NN. Statistical analysis: JW. Manuscript preparation: JW. Manuscript editing: NN, CJ, PH. Manuscript review: All authors.

\section{Acknowledgments}

All authors thank Nintita Sripaiboonkij Thokanit, DrPH for statistical analysis. Nuttapong Ngamphaiboon acknowledges a Research Development Grant from Ramathibodi Hospital.

The study was performed according to the principles of the 1964 Declaration of Helsinki. The Ramathibodi Ethics Committee approved the study (ID - COA. MURA2019/722).

\section{Availability of data}

The datasets used and/or analysed during the current study available from the corresponding author on reasonable request.

\section{Conflict of Interest}

All authors declare no conflict of interest.

\section{References}

Appropriate body-mass index for Asian populations and its implications for policy and intervention strategies (2004). Lancet, doi:10.1016/s0140-6736(03)15268-3.

Bedenne L, Michel P, Bouche O, et al (2007). Chemoradiation followed by surgery compared with chemoradiation alone in squamous cancer of the esophagus: FFCD 9102. J Clin Oncol, doi:10.1200/jco.2005.04.7118.

Bozzetti F, Migliavacca S, Scotti A, et al (1982). Impact of cancer, type, site, stage and treatment on the nutritiona status of patients. Ann Surg, 196, 170.

Cheng Y, Wang N, Wang K, et al (2012). Prognostic value of body mass index for patients undergoing esophagectomy for esophageal squamous cell carcinoma. Jpn J Clin Oncol, 
43, 146-53.

Conti S, West J, Fitzpatrick H (1977). Mortality and morbidity after esophagogastrectomy for cancer of the esophagus and cardia. Am Surg, 43, 92-6.

Cools-Lartigue J, Spicer J, Ferri LE (2015). Current status of management of malignant disease: Current Management of Esophageal Cancer. J Gastrointest Surg, 19, 964-72.

Cooper JS, Guo MD, Herskovic A, et al (1999). Chemoradiotherapy of locally advanced esophageal cancer long-term follow-up of a prospective randomized trial (RTOG 85-01). JAMA, doi:10.1001/jama.281.17.1623.

Crehange G, Bosset M, Lorchel F, et al (2006). Tumor volume as outcome determinant in patients treated with chemoradiation for locally advanced esophageal cancer. Am J Clin Oncol, doi:10.1097/01.coc.0000242346.25229.48.

Daly JM, Fry WA, Little AG, et al (2000) Esophageal cancer: results of an American College of Surgeons patient care evaluation study. J Am Coll Surg, 190, 562-72.

Di Fiore F, Lecleire S, Rigal O, et al (2006). Predictive factors of survival in patients treated with definitive chemoradiotherapy for squamous cell esophageal carcinoma. World J Gastroenterol, 12, 4185.

Duan X-F, Tang P, Shang X-B, et al (2017). High body mass index worsens survival in patients with esophageal squamous cell carcinoma after esophagectomy. Dig Surg, 34, 319-27.

Gu WS, Fang WZ, Liu CY, et al (2019). Prognostic significance of combined pretreatment body mass index (BMI) and BMI loss in patients with esophageal cancer. Cancer Manag Res, doi:10.2147/cmar.S197820.

Huhmann MB, Cunningham RS (2005). Importance of nutritional screening in treatment of cancer-related weight loss. Lancet Oncol, doi:10.1016/s1470-2045(05)70170-4.

Jemal A, Bray F, Center MM, et al (2011). Global cancer statistics. CA Cancer J Clin, 61, 69-90.

Jiang N, Zhao JZ, Chen XC, et al (2014). Clinical determinants of weight loss in patients with esophageal carcinoma during radiotherapy: a prospective longitudinal view. Asian Pac J Cancer Prev, doi:10.7314/apjcp.2014.15.5.1943.

Khan N, Bangash A, Sadiq M (2010) Prognostic indicators of surgery for esophageal cancer: a 5 year experience. Saudi $J$ Gastroenterol, doi:10.4103/1319-3767.70607.

Larrea J, Vega S, Martinez T, et al (1992). The nutritional status and immunological situation of cancer patients. Nutr Hosp, 7, 178-84.

Liu Z, Shi H, Chen L (2019). Prognostic role of pre-treatment C-reactive protein/albumin ratio in esophageal cancer: a meta-analysis. BMC Cancer, doi:10.1186/s12885-019. 6373-y.

Minsky BD, Pajak TF, Ginsberg RJ, et al (2002). INT 0123 (Radiation Therapy Oncology Group 94-05) phase III trial of combined-modality therapy for esophageal cancer: highdose versus standard-dose radiation therapy. J Clin Oncol, doi:10.1200/jco.2002.20.5.1167.

Nakatani M, Migita K, Matsumoto S, et al (2017). Prognostic significance of the prognostic nutritional index in esophageal cancer patients undergoing neoadjuvant chemotherapy. Dis Esophagus, doi:10.1093/dote/dox020s.

National Cancer Institute Doms, Ministryof Public Health, Thailand (2018). Hospital Based Cancer Registry 2017.

Saito T, Zeze K, Kuwahara A, Miyahara M, Kobayashi M (1990). Correlations between preoperative malnutrition and septic complications of esophageal cancer surgery. Nutrition, $\mathbf{6}$, 303-8.

SERVICES USDOHAH (November 27, 2017) Common Terminology Criteria for Adverse Events (CTCAE) v5.0.

Shapiro J, van Lanschot JJB, Hulshof M, et al (2015). Neoadjuvant chemoradiotherapy plus surgery versus surgery alone for oesophageal or junctional cancer (CROSS): longterm results of a randomised controlled trial. Lancet Oncol, doi:10.1016/s1470-2045(15)00040-6.

Shen LJ, Chen C, Li BF, Gao J, Xia YF (2013). High weight loss during radiation treatment changes the prognosis in under-/ normal weight nasopharyngeal carcinoma patients for the worse: a retrospective analysis of 2433 cases. PLoS One, doi:10.1371/journal.pone.0068660.

Sun L, Zhang H, Wu K (2014). Esophageal cancer: current options for therapeutic management. Gastrointest Tumors, 2014, 105-13.

Tamtai A, Jiarpinitnun C, Hiranyatheb P, et al (2017). Tolerability and efficacy of concurrent chemoradiotherapy comparing carboplatin/paclitaxel versus platinum/5-FU regimen for locally advanced esophageal and esophagogastric junction cancers. Med Oncol, doi:10.1007/s12032-017-1017-z.

Tepper J, Krasna MJ, Niedzwiecki D, et al (2008). Phase III trial of trimodality therapy with cisplatin, fluorouracil, radiotherapy, and surgery compared with surgery alone for esophageal cancer: CALGB 9781. J Clin Oncol, doi:10.1200/jco.2007.12.9593.

Torre LA, Bray F, Siegel RL, et al (2015). Global cancer statistics, 2012. CA Cancer J Clin, 65, 87-108.

van der Schaaf MK, Tilanus HW, van Lanschot JJ, et al (2014). The influence of preoperative weight loss on the postoperative course after esophageal cancer resection. J Thorac Cardiovasc Surg, 147, 490-5.

Wu N, Chen G, Hu H, Pang L, Chen Z (2015). Low pretherapeutic serum albumin as a risk factor for poor outcome in esophageal squamous cell carcinomas. Nutr Cancer, doi:10.1080/01635581.2015.1004726.

Yu X-L, Yang J, Chen T, et al (2018). Excessive pretreatment weight loss is a risk factor for the survival outcome of esophageal carcinoma patients undergoing radical surgery and postoperative adjuvant chemotherapy. Can J Gastroenterol Hepatol, doi:10.1155/2018/6075207.

Zhang L, Su Y, Chen Z, et al (2017). The prognostic value of preoperative inflammation-based prognostic scores and nutritional status for overall survival in resected patients with nonmetastatic Siewert type II/III adenocarcinoma of esophagogastric junction. Medicine (Baltimore), doi:10.1097/md.0000000000007647.

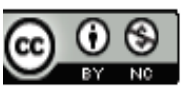

This work is licensed under a Creative Commons AttributionNon Commercial 4.0 International License. 